Ángulo R., Bayona, J., \& Esparza, M. (2014). Estrés laboral en el sector servicios. Revista Lebret (6). Bucaramanga, Colombia: Universidad Santo Tomás. pp. 351366. ISSN 2145-5996.

\title{
Estrés laboral en el sector servicios*
}

\section{Work stress in the services sector}

Rosalba Angulo Rincón ${ }^{l}$ Janeth Bayona Quiñonez Marnelly Esparza Durán

\begin{abstract}
Resumen
La presente investigación evalúa la respuesta de estrés laboral en 149 colaboradores pertenecientes a las tres sedes de una empresa de mensajería del Oriente colombiano. Los resultados muestran un nivel moderado de estrés laboral con presencia de síntomas fisiológicos, principalmente asociados con problemas gastrointestinales, no hallándose diferencias en función del género, ni del estado civil. Con relación a las variables organizacionales, se encontró una correlación estadísticamente significativa entre el número de horas laboradas por semana y nivel de estrés, a diferencia de las variables nivel jerárquico y sede de trabajo, en las que no se halló dicha correlación.
\end{abstract}

\section{Palabras clave}

Estrés laboral, psicología del trabajo y de las organizaciones, carga laboral, síntomas fisiológicos.

\section{Códigos de clasificación JEL: M12, M54}

\begin{abstract}
This research evaluates the response of job stress in 149 employees from the three offices of a courier company in eastern Colombia. The results show a moderate level of work stress with the presence of physiological symptoms, mainly associated to gastrointestinal problems, finding no differences in gender or marital status. Regarding organizational variables, a statistically significant correlation between the number of hours worked per week and stress level was found, unlike the hierarchical level variables and work place, where there was no correlation.
\end{abstract}

\section{Keywords}

Job Stress, industrial and organizational psychology, workload, physiological symptoms

\footnotetext{
* De la investigación "Evaluación de síntomas asociados al estrés laboral en una empresa de mensajería del Oriente colombiano". Pontificia Universidad Javeriana y Subcentro de Seguridad Social y Riesgos Profesionales.

1 Doctora en Psicopatología Infantil, del Adolescente y del Adulto, magíster en Administración de Empresas. Docente de la Universidad Pontificia Bolivariana, Colombia. Correo electrónico: rosalba.angulo@upb.edu.co

$2 \quad$ Psicóloga. Correo electrónico: janeth671@hotmail.com

3 Psicóloga. Correo electrónico: marnelly_esparza@hotmail.com
} 


\section{Introducción}

El estrés representa uno de los problemas de salud más graves en la actualidad debido a sus consecuencias físicas, emocionales, cognoscitivas y conductuales, que pueden desencadenar enfermedades incapacitantes en el individuo, afectar procesos de trabajo y la calidad de los servicios que se brindan en una institución. Es sabido que la sobre exposición a aquellos factores que resultan estresantes para las personas, ocasiona un desgaste y agotamiento de los recursos de afrontamiento y adaptación, lo que a su vez repercute en la salud física, psicológica y emocional de la persona.

Este concepto es importante y tiene relevancia en el ámbito laboral porque el individuo puede responder de diferentes maneras ante un suceso inesperado; así para algunas personas una situación puede ser muy amenazante, pero para otras esa misma situación podría pasar desapercibida. En este sentido, Díaz (2011) señala que dichas respuestas pueden activar mecanismos fisiopatológicos de una enfermedad.

Además,

El estrés laboral es consecuencia de múltiples factores causales, incluyendo el ambiente físico, el trastorno de las funciones biológicas, la carga laboral, el contenido y la organización del trabajo, en interacción con todos aquellos componentes o dimensiones de la vida de cada individuo que no dependen necesariamente del trabajo (García, 2006, p. 6);

Es decir que este se produce cuando las demandas del trabajo rebasan la capacidad de afrontamiento del individuo.

Al respecto, el modelo de estrés de Lazarus y Folkman (1986) destaca la importancia de la percepción adecuada de la competencia, pues en la medida que la persona perciba que es capaz de afrontar determinada situación, el estrés disminuirá, mientras si el trabajador considera que la tarea sobrepasa sus capacidades, podría sobrevenir el estrés, ya que en su valoración secundaria se determinaría que no se poseen los recursos adecuados para llevar a cabo su labor. Sin embargo, junto a la motivación existen otros factores que pueden resultar protectores del estrés laboral, como el clima organizacional óptimo, la legislación laboral, el liderazgo, la percepción de la calidad de vida en relación con la dimensión ocupacional y otras variables de orden subjetivo como el proyecto de vida, el significado del trabajo para los empleados, la satisfacción laboral y personal. En este sentido, Keeton, Fenner, Johnson y Hayward (2007) sugieren que la empresa debe promover la articulación del trabajo con la vida del empleado, de tal modo que haga parte de las actividades para las cuales él es competente, se percibe como tal y en la medida de lo posible, disfrute realizar.

Una serie de cambios introducidos en el mundo del trabajo, particularmente referidos a la globalización, las nuevas formas de direccionamiento, la incertidumbre 
producto de las nuevas modalidades de contratación, la migración laboral, los altos estándares de competencia y desempeño, entre otros. (OIT, 2006; Smith, 1997) han planteado nuevos desafíos para las organizaciones como para cada uno de los trabajadores, por lo cual, el trabajo se ha convertido en una fuente de estrés (Maslach, 2009). Como consecuencia de estos cambios y de las repercusiones que han tenido sobre los trabajadores, distintas organizaciones internacionales han creado normas y códigos que pretenden regular e intervenir las condiciones de las empresas, y las exhorta a establecer estrategias para garantizar el bienestar de sus empleados. Uno de los mayores problemas a los que se enfrentan las organizaciones es a la presencia de estrés laboral (y en su mayor expresión el síndrome de burnout) el cual disminuye el desempeño de sus trabajadores (Fernández, 2010), e impacta negativamente el clima de la organización (Quintero, Africano \& Faría, 2008).

Como puede verse, en los últimos años la importancia del ambiente psicosocial en los lugares de trabajo ha adquirido una mayor relevancia, ya que como plantea la Organización Internacional del Trabajo (OIT, 2006) el desarrollo tecnológico, económico, el incremento de la productividad, entre otros no solo dependen de los diferentes medios de producción o del incremento de maquinarias y nueva tecnología, sino también del bienestar y la salud de los trabajadores y de sus familias, pues son ellos los que desempeñan algunas de las labores más importantes en las organizaciones. Todo esto implica la acción preventiva y la intervención, en donde se tienen en cuenta, no solo los riesgos físicos que se pueden llegar a presentar, sino también los diversos factores psicosociales conexos a las organizaciones, los cuales pueden llegar a tener gran influencia sobre el bienestar físico y emocional de los trabajadores. El argumento de base que justifica la intervención de la psicología en torno al bienestar laboral es que "todos los problemas psicosociales y de salud relacionados con el trabajo son por definición susceptibles de ser prevenidos, al estar estos enmarcados en las condiciones laborales, que en últimas son construcciones sociales, creaciones humanas entes artificiales que pueden ser modificados" (Benavides, Ruiz y García, 2000 citado por Andrade y Gómez, 2008, p. 13).

La promulgación en Colombia del Plan Nacional de Salud Ocupacional 20032007, se planteó que todos los actores del sistema: trabajadores, empleadores, asegurados y el Gobierno nacional, se deben comprometer con fortalecer la promoción de la seguridad social y salud en el trabajo, fomentar una cultura del autocuidado y la prevención de los riesgos profesionales (Ministerio de la Protección Social, 2004 citado por Peña, Colina y Vásquez, 2009, p. 58).

De lo anterior, se desprende que en las organizaciones es preciso realizar una aproximación orientada a la consecución y mantenimiento del bienestar personal que, al mismo tiempo, busque mejorar la calidad de vida y el bienestar laboral de los trabajadores (González y Garrosa, 2007 citado por, Extremadura, Durán y Rey, 
2010). Cuando las organizaciones ofrecen a sus trabajadores un ambiente laboral apropiado, están coadyuvando al mejoramiento de la calidad de vida del talento humano, que se traduce en menores problemas de salud, familiares y de convivencia.

El Ministerio de la Protección Social (2004) indica que para el caso del régimen contributivo en Colombia se presentan 26.5 casos de diagnóstico de enfermedad profesional por cada 100000 habitantes afiliados al sistema y 22 casos por cada 100000 trabajadores cotizantes del sistema general de riesgos profesionales. También se ha encontrado que las enfermedades profesionales son sub-diagnosticadas y que las enfermedades mentales que tienen origen laboral no son mencionadas en el informe sobre enfermedades laborales, también señala que uno de cada 3 trabajadores colombianos están expuestos a factores de riesgos psicosociales durante la jornada laboral completa y entre un 20 a un 33\% manifestaron sentir altos niveles de estrés (Ministerio de la Protección Social, 2009). Por tanto, a través de la promulgación del Decreto 2566 del 2009 se ha reconocido el estrés laboral como una enfermedad y una respuesta adaptativa mediada por características individuales o procesos psicológicos que deriva en ansiedad y trastornos psiquiátricos como el síndrome de burnout (Díaz, 2011).

De este modo, la evaluación del estrés laboral es clave para la gestión del talento humano y de la responsabilidad que actualmente tienen las organizaciones con sus trabajadores. Para responder a esta necesidad, desde la psicología organizacional se han desarrollado instrumentos de medición del estrés, protocolos de evaluación y programas de prevención e intervención. En consecuencia, es claro que el primer paso en la intervención del estrés es la evaluación para la obtención de un diagnóstico completo, en procura de generar programas que satisfagan las necesidades puntuales de las empresas. Así mismo, debe considerarse que la medición del estrés no es una variable aislada, sino una parte integral de la evaluación de los factores psicosociales que en una relación dinámica, influyen en la salud y el desempeño laboral de las personas.

Los factores de estrés pueden evaluarse en distintas dimensiones: conductuales, cognitivas, emocionales y sociales (Pontificia Universidad Javeriana \& Subcentro de Seguridad Social y Riesgos Profesionales, 2010) y en relación con variables sociodemográficas (Schaufeli, 1999) y organizacionales (Tejada \& Gómez, 2009). Concretamente en el departamento de Santander, los estudios sobre estrés laboral son pocos, casi inexistentes, lo cual resulta preocupante dada la alta prevalencia de este fenómeno a nivel mundial y la necesidad de dar cumplimiento a la nueva normatividad laboral colombiana, en materia de salud ocupacional y riesgos 
laborales. En tal sentido, la evaluación de los síntomas asociados con el estrés laboral en nuestro contexto constituye un valioso aporte, además si se considera la tendencia de la investigación en nuestro país de orientarse al estudio del tema en el sector de la salud, sin abordar otros sectores como el de servicios.

Así las cosas, el trabajo que aquí se presenta evaluó la respuesta de estrés de un grupo de colaboradores en una empresa de mensajería, líder en la región que brinda soluciones a sus clientes en la recolección, transporte, almacenamiento y distribución de documentos y mercancías. Dicha organización contempla dentro de sus metas el cuidado y mejora del talento humano, para lo cual apoya la investigación y evaluación del malestar psicológico asociado al trabajo que permita diseñar estrategias que favorezcan la salud mental y el desarrollo integral sus trabajadores.

\section{Método}

\section{Tipo de estudio}

El trabajo corresponde a un estudio cuantitativo de corte transversal, con un diseño correlacional descriptivo.

\section{Participantes}

La población diana está conformada por 312 trabajadores (en adelante colaboradores) de la empresa de mensajería, pertenecientes a las 3 zonas de la organización. Los criterios de inclusión para la muestra fueron colaboradores con un año mínimo de vinculación a la empresa y que laboraran en una de las zonas de la Regional Oriente. De esta forma se trabajó con una muestra no probabilística conformada por 149 colaboradores de ambos sexos (hombres $n=123,83 \%$; mujeres $n=26,17 \%$ ). La media de edad es de 32.61 años y una desviación estándar de 8.0.

Con respecto a las condiciones de trabajo (tabla 1), un número total de 99 colaboradores (66\%) laboran en la zona de Bucaramanga, 35 en Barrancabermeja (24\%) y $15(10 \%)$ en la ciudad de Cúcuta. En cuanto al tipo de contrato $129(87 \%)$ es a término fijo y $20(13 \%)$ a término indefinido. En el nivel jerárquico, se evaluaron 22 colaboradores (15\%) con cargo administrativo y 127 (85\%) operarios o subalternos. Con relación a la carga horaria, un gran porcentaje trabaja 8 horas diarias $(n=105$, $71 \%$ ), aunque algunos laboran hasta 14 horas diarias. 
Tabla 1. Variables organizacionales de la muestra evaluada

\begin{tabular}{|l|l|c|c|}
\hline \multicolumn{2}{|c|}{ Variable } & Frecuencia & Porcentaje \\
\hline \multirow{3}{*}{ Sede } & Bucaramanga & 99 & 66 \\
\hline & Barrancabermeja & 35 & 24 \\
\hline \multirow{2}{*}{ Contrato } & Cúcuta & 15 & 10 \\
\hline \multirow{3}{*}{ Nivel jerárquico } & Fijo & 129 & 87 \\
\hline & Indefinido & 20 & 13 \\
\hline \multirow{3}{*}{ Carga horaria (diaria) } & Administrativo & 22 & 15 \\
\hline & Subalterno & 127 & 85 \\
\hline & 8 & 105 & 71 \\
\hline & 9 & 11 & 8 \\
\hline & 10 & 11 & 7 \\
\hline & 12 & 2 & 1 \\
\hline & 13 & 8 & 5 \\
\hline
\end{tabular}

Fuente: autores.

\section{Instrumentos}

Para la recolección de los datos socio-demográficos y laborales se diseñó un formato en el cual se registraron variables como edad, sexo y estado civil. Con relación a los datos laborales se consideraron algunas variables organizacionales como jerarquía, número de horas semanales de trabajo y sede de la regional a la cual estaba adscrito cada uno de los participantes.

Los síntomas asociados con el estrés fueron medidos mediante el Cuestionario para la evaluación del estrés tercera versión (Ministerio de la Protección Social, Pontificia Universidad Javeriana y Subcentro de Seguridad Social y Riesgos Profesionales, 2010). Este instrumento tiene como objetivo identificar los síntomas físiológicos, de comportamiento social, intelectual y emocional del estrés. Se diseñó con el fin de evaluar síntomas reveladores de reacciones de estrés, distribuidos en cuatro categorías principales (Franco y Ortíz, 2011), según el tipo de síntoma: a) fisiológicos (8 ítems), b) comportamiento social (4 ítems), c) intelectuales y laborales (10 ítems), d) emocionales (9 ítems). Los baremos (en centiles) fueron obtenidos de una muestra de 4521 trabajadores afiliados al Sistema de Riesgos Profesionales pertenecientes a las seis regiones de Colombia y a los cuatro grandes sectores económicos. La validez fue concurrente tomando la segunda versión del cuestionario y calculando su correlación con las escalas de salud general, vitalidad y salud mental del cuestionario SF-36v2, escalas validadas con anterioridad en el país. De igual manera se observó 
una correlación inversa y significativa entre los resultados del cuestionario de estrés y las tres escalas del SF-36v2: estado general de salud percibido, vitalidad y salud mental (Vilagut, et al., 2005). Mediante el Alfa de Cronbach fue posible calcular la consistencia interna, con un coeficiente de $0.889(\mathrm{p}=0.001)$ y aplicando el método test-retest, se obtuvo la confiabilidad total con un resultado significativo $(\mathrm{r}=0.851$; $\mathrm{p}=0.005)$.

\section{Procedimiento}

Tras haber obtenido la autorización por parte de la empresa para realizar la investigación, se dieron a conocer los propósitos de esta y la metodología empleada, la cual fue aceptada por los funcionarios responsables de la gerencia administrativa, quienes permitieron el acceso a la institución y a la base de datos del personal, de tal modo que se pudieran acordar los tiempos y horarios de aplicación de los cuestionarios. Además se informó a los participantes sobre el carácter estrictamente confidencial de la información y su derecho a renunciar al estudio en cualquier momento.

Los cuestionarios fueron diligenciados de manera voluntaria por cada colaborador, tras firmar el consentimiento informado. Luego se llevó a cabo la calificación de la prueba, tabulación de la información recolectada y procesamiento de la información mediante un análisis descriptivo, utilizando medidas de tendencia central (media) y medidas de variabilidad (error estándar de la media) para las puntuaciones alcanzadas en cada uno de los instrumentos de evaluación aplicados.

Finalmente, con el objetivo de establecer la relación entre los resultados del Cuestionario de Estrés y variables sociodemográficas y organizacionales, se empleó el test t para muestras relacionadas. Así mismo, un análisis de varianza de una vía para llevar a cabo una diferenciación entre grupos distribuidos según variables sociodemográficas (edad y sexo) y variables organizacionales (jerarquía, sede y carga horaria). El nivel de significancia establecido fue de $\mathrm{p}<0.05$.

\section{Resultados}

Al evaluar la respuesta de estrés laboral, mediante el Cuestionario de estrés laboral, tercera versión (Pontificia Universidad Javeriana y Subcentro de Seguridad Social y Riesgos Profesionales, 2010) se encontró que en promedio los trabajadores presentan sintomatología asociada con el estrés en un nivel medio (puntaje directo 8.9, desviación estándar 2.5; puntaje corregido 14.5, desviación estándar 4.03). El análisis de frecuencias arrojó que la sintomatología de estrés es media, seguida de los niveles bajo y muy bajo. Lo anterior significa que el $24 \%$ de la muestra reportó un nivel medio de estrés, seguido por una percepción baja y muy baja $(23 \%)$, siendo los niveles alto y muy alto los porcentajes menos significativos (17\% y $14 \%$ respectivamente). 
El análisis de los promedios por grupos de síntomas muestra que el mayor porcentaje de esta (57\%) puntúo con el valor más alto los síntomas físiológicos, seguido por los síntomas sociales (24\%), los intelectuales (18\%) y los emocionales $(5 \%)$. Estos valores se recogen en la tabla 2. En vista de que los síntomas fisiológicos fueron los que obtuvieron un mayor puntaje, se realizó el análisis de los promedios por ítem, hallándose que los síntomas asociados a problemas gastrointestinales son los más comunes.

Tabla 2. Puntajes promedio por pregunta en la dimensión de síntomas fisiológicos

\begin{tabular}{|l|c|}
\hline \multicolumn{1}{|c|}{ Ítem } & $\begin{array}{c}\text { Porcentaje } \\
\%\end{array}$ \\
\hline Dolores en el cuello y espalda o tensión muscular & 18 \\
\hline $\begin{array}{l}\text { Problemas gastrointestinales, úlcera péptica, acidez, problemas digestivos o del } \\
\text { colón. }\end{array}$ & 23 \\
\hline Problemas respiratorios & 14 \\
\hline Dolor de cabeza & 14 \\
\hline Trastornos del sueño como somnolencia durante el día o desvelo en la noche & 13 \\
\hline Palpitaciones en el pecho & 8 \\
\hline Cambios fuertes de apetito & 6 \\
\hline Problemas relacionados con la función órganos genitales (impotencia, frigidez). & 4 \\
\hline
\end{tabular}

Fuente: autor.

\section{Análisis de los resultados en el Cuestionario para la evaluación del estrés, tercera versión en función de variables sociodemográficas}

En relación con el análisis de comparación de medias (t de Student), se presentan en la tabla 3 los puntajes directos, puntajes corregidos y nivel de estrés. El análisis realizado indica que no existe una diferencia estadísticamente significativa en función del sexo.

Tabla 3. Comparación de resultados en el Cuestionario para la evaluación del estrés, tercera versión en relación con variables sociodemográficas

\begin{tabular}{|l|l|l|c|}
\hline \multicolumn{3}{|c|}{ Puntajes directos } \\
\hline & \multicolumn{2}{|c|}{ Promedio } & \multicolumn{2}{c|}{ EMM } & P \\
\hline Hombres & 10.30 & 0.59 \\
\hline Mujeres & 7.58 & 1.05 \\
\hline \multicolumn{3}{|c|}{ Puntajes corregidos } \\
\hline
\end{tabular}




\begin{tabular}{|l|l|l|l|}
\hline Hombres & 12.39 & 0.97 & 0.068 \\
\hline Mujeres & 16.85 & 1.71 & \\
\hline & \multicolumn{3}{|c|}{ Nivel de estrés } \\
\hline Hombres & 3 & 0.12 & 0.13 \\
\hline Mujeres & 3 & 0.25 & \\
\hline
\end{tabular}

Nota . Muestra total = 149 colaboradores; 123 hombres, 26 mujeres. Nivel de significancia $\mathrm{P}<0,05$.

Fuente: autores.

Así mismo, y con respecto al estado civil el análisis de varianza de una vía (Anova) indicó que no existe diferencia estadísticamente significativa entre los grupos evaluados en ninguno de los puntajes obtenidos (puntaje directo, $\mathrm{P}=0,18$ y nivel de estrés, $\mathrm{P}=0,19$ ).

\section{Análisis de los resultados en el Cuestionario para la evaluación del estrés en relación con variables de la organización}

Con el propósito de analizar si existían diferencias entre los niveles de jerarquía (administrativos $(\mathrm{N}=22)$ y subalternos $(\mathrm{N}=127)$, se llevó a cabo un test de comparación de media directos (puntajes directos $\mathrm{P}=0,14$ corregidos $\mathrm{P}=0,14$, nivel de estrés $\mathrm{P}=0,19$ ). Dicho análisis igualmente se aplicó para establecer la diferencia entre los colaboradores con contrato a término fijo y contrato indefinido, no hallándose en ambos caso, un índice estadísticamente significativo (puntajes directos $\mathrm{P}=0,97$, corregidos $\mathrm{P}=0,97$ y nivel de estrés $\mathrm{P}=0,96$.

Por otro lado, la tabla 4 presenta los resultados obtenidos del análisis de varianza de una vía (Anova), que permiten señalar que no existe una diferencia estadísticamente significativa entre sedes y carga horaria.

Tabla 4. Comparación de resultados en el Cuestionario para la evaluación del estrés, tercera versión en relación con variables de la organización

\begin{tabular}{|l|c|c|c|}
\hline & Puntaje directo & Puntaje corregido & Nivel de estrés \\
\hline Carga horaria diaria & 0.07 & 0.07 & 0.90 \\
\hline Sedes & 0.22 & 0.22 & 0.20 \\
\hline
\end{tabular}

Nota. Nivel de significancia $\mathrm{P}<0,05$.

Fuente: autores. 
Para las sedes a las que pertenecen los colaboradores (Bucaramanga, Barrancabermeja, Cúcuta) y la carga horaria diaria $(8,9,10,11,12,13$ y 14 horas) se realizó un análisis de varianza de una vía (Anova), a través del cual no se encontraron diferencias estadísticamente significativas.

Finalmente, al establecer una correlación de Spearman entre el número de horas laboradas y el puntaje de estrés, se halló una correlación positiva entre estas variables (Puntajes directos $\mathrm{P}=0.01$; puntajes corregidos $\mathrm{P}=0.01$; nivel de estrés $\mathrm{P}=0.01$ ).

\section{Discusión}

El presente estudio evaluó la respuesta de estrés laboral en una empresa de mensajería del nororiente colombiano. Los resultados obtenidos revelaron que los colaboradores perciben un nivel medio de sintomatología asociada al estrés, lo cual sugiere que la capacidad de afrontamiento a factores estresores de los colaboradores de la empresa les permite ubicarse en el límite de soporte de su propia respuesta ante contextos amenazantes. Al realizar un análisis del factor que mayor puntaje obtuvo en el cuestionario se encontró que las variables fisiológicas fueron las más frecuentes. Estos resultados son consistentes con otras investigaciones en donde los síntomas somáticos del estrés son los más identificados y por los cuales las personas reportan sentirse más cansadas (Alkhader, 2006). En este sentido Barragan-Solís (2000) destaca que la sintomatología física puede conducir a otros tipos de malestar que inciden en el comportamiento de las personas, aumentando su irritabilidad, así como su percepción del cansancio y el agotamiento. De ahí la importancia de detectar los factores de riesgo psicosocial (intra y extra laborales) que pueden estar relacionados con los efectos identificados (Ministerio de la Protección Social, Pontificia Universidad Javeriana y Subcentro de Seguridad Social y Riesgos Profesionales, 2010) y la necesidad de emprender acciones sistemáticas de intervención para prevenir efectos perjudiciales en la salud.

Por otro lado, y contrario a lo propuesto en la literatura (Isis Internacional, 2001; Roxburg, 1996; Trucco, 1999) acerca del estrés laboral en función del sexo, no se encontraron diferencias estadísticamente significativas entre los grupos. No obstante, un estudio sugiere que debido a los cambios en la perspectiva de género y a la desaparición paulatina de los roles tradicionales, las respuestas de estrés en relación con el género se han hecho cada vez menos notables (Aldrete, Pando, Aranda y Torres, 2006). Sin embargo, Torkelson y Muhonen (2004) hallaron diferencias entre hombres y mujeres, lo cual fue atribuido al temor de estas últimas de perder el empleo o de disminuir su desempeño laboral, constituyéndose en un factor que aumenta la percepción de los niveles de estrés. En cargos de menor jerarquía, algunos autores (Catalina, Corrales, Cortés y Gelpi, 2008) han propuesto que debido al acceso limitado a la educación, se siguen manteniendo los roles de género tradicionales, por lo cual la carga de los oficios del hogar podría resultar 
perjudicial para las trabajadoras, aumentando las posibilidades de presencia de síntomas de estrés. Otro factor que ha sido analizado es la maternidad/paternidad, así en un estudio realizado en una empresa de producción de alta demanda se encontró que dependiendo de la edad de los hijos, su presencia puede constituirse en un factor protector o predisponente del estrés; de esta forma, los trabajadores con hijos entre los 0 y 6 años presentaban mayores síntomas de estrés que los empleados con hijos adolescentes. Por otro lado, contar con hijos adultos sí actuaba como factor protector (Marrero, Aguilera y Aldrete, 2008).

Con respecto al estado civil, tampoco se observaron diferencias estadísticamente significativas, hallazgo que está en contraposición a los obtenidos en otras investigaciones, las cuales sugieren que la red inmediata de apoyo constituye un factor determinante para la percepción del estrés laboral (Luceño, García, Jaén y Díaz, 2005; Schaufeli, 1999). Aquí es importante resaltar que para la evaluación de la red de apoyo y factores psicosociales de la familia es necesario considerar que no en todos los casos la presencia de la familia constituye una fuente de apoyo o de ayuda para el afrontamiento y el manejo del estrés. De acuerdo con Durán (2010), en ocasiones los grupos de trabajadores que reportan mayores niveles de estrés son aquellos que refieren presentar problemas familiares o de relación, de modo que sería necesario determinar cuáles son las variables de la organización y los factores extrínsecos que contribuyen con el estrés, para así realizar una intervención directa mediante la implementación de los programas de bienestar laboral y responsabilidad social existentes en las empresas (Goiria, San Sebastián y Torres, 2003).

En cuanto a las variables relacionadas con la organización, no se hallaron diferencias respecto al nivel de jerarquía, lo cual sugiere que los administrativos y los subalternos perciben el mismo nivel de estrés independientemente de esta variable. No obstante, algunos estudios (Naranjo y González, 2012) han indicado que en los cargos de alta gerencia los trabajadores se sienten más presionados, en especial aquellos que ejecutan cargos intermedios, pues deben administrar personal y responder por los resultados de su equipo de trabajo, mientras que los subalternos al conocer que la responsabilidad inmediata no recae directamente sobre ellos, perciben un menor nivel de estrés. Sin embargo, otro estudio señala que los cargos administrativos son puestos de menor estrés en la medida en que se relacionan con mayores garantías y con factores de recompensa, tales como posibilidades de ascenso y reconocimiento, además de promover más asiduamente la constante preparación de los trabajadores, lo que resulta un factor motivacional (De los Ríos, Ocampo, Landeros, Ávila y Tristán, 2007). Respecto a este último aspecto, algunas investigaciones han hallado resultados que apoyan la idea de que la motivación es clave para el manejo del estrés laboral debido a que favorece que los trabajadores se perciban como competentes y capaces para hacerle frente a las situaciones problemáticas que se presentan en su contexto laboral (Fernet, 2013). 
Lo anterior tiene sentido si se tiene en cuenta que de acuerdo con la teoría del estrés de Lazarus y Folkman (1986) el distrés sobreviene cuando en la evaluación secundaria la persona determina que no posee los recursos suficientes para solucionar un problema; claro que debe recordarse que en este punto, las personas ya han pasado la fase de agotamiento, la cual ocurre únicamente después de haber estado expuesta por un periodo de tiempo prolongado a la fuente de estrés (factor). En el caso del estrés (distrés) laboral los factores pueden estar relacionados con variables externas o internas a la organización, mientras en el caso de las variables internas la carga laboral, las demandas bajo presión, la carga horaria y las relaciones interpersonales, así como el apoyo de los jefes pueden ser factores que mejoran o empeoran la percepción de los niveles de estrés. Las variables organizacionales se relacionan directamente con las demandas del trabajo (cuantitativas, emocionales, de carga mental, responsabilidad, esfuerzo físico, jornada laboral, rol asumido), el control sobre el trabajo (autonomía, oportunidades de desarrollo y uso de habilidades y destrezas, claridad del rol y capacitación), el liderazgo, las relaciones interpersonales y la recompensa (Pontificia Universidad Javeriana y Subcentro de Seguridad Social y Riesgos Profesionales, 2010).

Sobre el particular, la variable carga horaria de trabajo se correlacionó positivamente con el nivel percibido en la muestra evaluada, coincidiendo con el estudio realizado por Leka, Griffiths y Cox (2004). Dado el tipo de empresa en la cual se accedió, podría hipotetizarse que los largos turnos son especialmente agotadores, debido al trabajo que deben realizar los colaboradores, además los turnos de duración prolongada han correlacionado en otros estudios con presencia de síntomas físicos, producto de las posturas inadecuadas, los movimientos repetitivos y la gran cantidad de atención activa que requieren algunas tareas (Muñoz, 2010).

A pesar de estos hallazgos, algunas investigaciones han encontrado que la variable carga horaria no es un factor que module la percepción del estrés, sino que su nivel se encuentra asociado con la tarea que deba realizarse en el turno (Cruz et al., 2010). Por otra parte, debe considerarse que en algunas organizaciones existe un sistema de compensación que permite largos periodos de descanso luego de los turnos con una carga laboral extendida. Según Díaz y Feldman (2008) los trabajadores pueden ver en esos días libres una recompensa por el trabajo de modo que se sienten motivados hacia el trabajo, reduciendo la presencia de estrés. Esta última idea coincide con el sistema de carga horaria de la empresa evaluada, por lo cual sería una variable de interés para estudiar en futuras investigaciones. Cabe señalar que este estudio realiza una exploración de los síntomas asociados al estrés en una empresa que pertenece al sector de servicios que poco ha sido relacionado con el estrés laboral. Los resultados obtenidos en esta investigación constituyen una aproximación en el contexto santandereano que podría orientar la creación de protocolos de intervención de la sintomatología de estrés relacionado con el trabajo, siendo para el caso puntual, el manejo de los síntomas físicos del estrés. 
Con respecto a la correspondencia estrés laboral y variables sociodemográficas, se señala que no existen diferencias estadísticamente significativas en los puntajes promedio entre hombres y mujeres y tampoco en los grupos por estado civil. Este resultado indica que dichas variables no interfieren en la respuesta de estrés laboral en este grupo de trabajadores. En atención a las variables de la organización, se halló que al comparar los grupos por nivel jerárquico y sede de trabajo, no se observan diferencias estadísticamente significativas en el nivel de estrés, lo que indica que dichas variables no modulan la respuesta de estrés en este grupo de trabajadores.

Finalmente, el análisis de correlación entre horas trabajadas y nivel de estrés indicó que existe una correlación positiva entre horas de trabajo diarias y el nivel de estrés laboral. Este resultado sugiere que los trabajadores perciben mayor estrés laboral cuanto más tiempo deban trabajar, sugiriendo que las jornadas largas de trabajo pueden provocar que se genere la respuesta de estrés.

\section{Referencias}

Aldrete, M., Pando, M., Aranda, C., y Torres, T. (2006). Acoso psicológico en el trabajo: ¿un problema de género? Enseñanza e Investigación en Psicología, 11(1), 53-63.

Alkhader, O. (2006). Assesing occupational stress, strain and coping for North American teachers in Kuwait. Psychological Reports, 99(3), 681-690.

Andrade, V., y Gómez, I. C. (2008). Salud laboral. Investigaciones realizadas en Colombia. Pensamiento Psicológico, 4(10), 9-25.

Barrangan-Solís, A. (2000). La salud y el ambiente laboral entre los anestesiólogos. Contexto social y laboral. Revista de Medicina del Hospital General, 63(4), 247253.

Catalina, C., Corrales, H., Cortés, M., y Gelpi, J. (2008). Las diferencias de género en el nivel de exposición a riesgos psicosociales entre trabajadores/as de la comunidad autónoma de Madrid. Resultados preliminares del plan de investigación del estrés laboral de Ibermutuamur. EduPsykhé, 7(2), 185-200.

Cruz, M., Chaves, M., Barcellos, R., da Silva, L., de Oliveira, I. y Pedrão, L. (2010). Exceso de trabajo y agravios mentales a los trabajadores de la salud. Revista Cubana de Enfermería, 26(1), 52-64.

De los Ríos, J., Ocampo, M., Landeros, M., Ávila, M., y Tristán, T. (2007). Burnout en personal administrativo de una dependencia gubernamental: Análisis de sus factores relacionados. Investigación y Ciencia, 37, 26-34.

Díaz, A. y Feldman, L. (2008). Validación preliminar del cuestionario de estrés laboral (desbalance-esfuerzo/recompensa) en una muestra de trabajadores de la 
salud venezolanos. Ponencia presentada en el Segundo Foro de las Américas en Investigación sobre Factores Psicosociales. Jalisco: Universidad de Guadalajara.

Díaz, D. (2011). Estrés laboral y sus factores de riesgo psicosocial. Revista CES Salud Pública, 2(1), 80-84.

Durán, M. (2010). Bienestar psicológico: El estrés y la calidad de vida en el contexto laboral. Revista Nacional de Administración, 1(1), 71-84.

Extremadura, N., Durán, A. y Rey, L. (2010). Recursos personales, síndrome de estar quemado por el trabajo y sintomatología asociada al estrés en docentes de enseñanza primaria y secundaria. Ansiedad y Estrés, 16(1), 47-60.

Franco, O. L., y Ortíz, O. L.(2011). Síndrome de Burnouty estrés en médicos estudiantes de Salud Ocupacional. Repositorio Universidad del Rosario. Recuperado de: http://repository.urosario.edu.co/bitstream/handle/10336/2845/79791373-012. pdf? sequence $=1$

Fernández, H. (2010). La influencia de los factores estresantes del trabajo en el rendimiento laboral. Invenio 13(25), 111-124.

Fernet, C. (2013). The role of work motivation in psychological health. Canadian Psychology, 54(1), 72-74.

García, C. A. (2006). Estrés laboral en personal de la Unidad de Emergencia Ginecoobstétrica. (Trabajo de grado). Hospital Clínico Regional Valdivia. Facultad de Medicina, Universidad Austral de Chile.

Goiria, J., San Sebastián, X., y Torres, E. (2003). Estudio de evaluación e intervención del estrés laboral en la policía municipal. Revista de la Sociedad Española de Salud Laboral en la Administración Pública, 1(7), 5-8.

Isis Internacional. (2001). El estrés. Agenda Salud, 24, 2-6.

Jaramillo, V., y Gómez, I. (2008). Salud laboral. Investigaciones realizadas en Colombia. Pensamiento Psicológico, 4, 9-25.

Keeton, K., Fenner, D., Johnson, T., y Hayward, R. (2007). Predictors of physician career satisfaction, work-life balance, and burnout. Obstetrics \& Gynecology, 109(4), 949-955.

Lazarus, R., y Folkman, S. (1986). Estrés y procesos cognitivos. Barcelona: Martínez Roca.

Leka, S., Griffiths, A., y Cox, T. (2004). La organización del trabajo y el estrés. Reino Unido: Organización Mundial de la Salud.

Luceño, L., García, J., Jaén, M., y Díaz, E. (2005). Evaluación de factores psicosociales en el entorno laboral. EduPsykhé, 4(1), 19-42. 
Marrero, M., Aguilera, M., y Aldrete, M. (2008). Estrés psicosocial laboral en embarazadas del sector de la salud de ciudad de La Habana, Cuba 2005-2006. Revista Peruana de Medicina Experimental y Salud Pública, 25(2), 200-203.

Maslach, C. (2009). Comprendiendo el burnout. Ciencia y Trabajo, 11(32), 37-43.

Ministerio de la Protección Social. (2004). Informe de la enfermedad profesional en Colombia. (En línea). http://www.minproteccionsocial.gov.co/actualizado.

Ministerio de la Protección Social. (2009). Guía técnica para el análisis de exposición a factores de riesgo ocupacional en el proceso de evaluación para la calificación de origen de la enfermedad profesional. Bogotá: El Ministerio.

Muñoz, G. (2010). Estrés laboral y factores ergonómicos como determinantes de dolor musculo esquelético en diversas zonas corporales. Trabajo libre presentado en el Tercer Foro de las Américas sobre Factores Psicosociales. Cuernavaca: Red de Investigadores sobre Factores Psicosociales en el Trabajo.

Naranjo, R., y González, M. (2012). Habilidades gerenciales del líder en las medianas empresas de la región caribe colombiana. TEACS, 10, 25-36.

Oficina Internacional del Trabajo-OIT. (2006). Cambios en el mundo del trabajo. Conferencia Internacional del Trabajo, 95ª reunión 2006. Informe I (C) (En línea). http://www.ilo.org/public/spanish/stan-dards/relm/ilc/ilc95/pdf/rep-i-c.pdf

Peña, E., Colina, E., y Vásquez, A. (2009). Actividad Física en empleados de la Universidad de Caldas, Colombia. Hacia la Promoción de la Salud, 14, 52-65.

Pontificia Universidad Javeriana y Subcentro de Seguridad Social y Riesgos Profesionales. (2010). Batería de instrumentos para la evaluación de factores de riesgo psicosocial. Bogotá D.E.: Ministerio de la Protección Social.

Quintero, Africano y Faría. (2008). Clima organizacional y desempeño laboral del personal de la empresa Vigilantes Asociados Costa del Lago. Negotium, 9, 33-51.

Roxburg, S. (1996). Gender differences in workand well-being: effects of exposure and vulnerability. Journal of Health and Social Behavior, 37, 265-277.

Schaufeli, W. (1999). Evaluación de riesgos psicosociales y prevención del estrés laboral: algunas experiencias holandesas. Revista de Psicología del Trabajo y de las Organizaciones, 15(2), 147-171.

Tejada, P., y Gómez, V. (2009). Factores psicosociales y laborales asociados al burnout de psiquiatras en Colombia. Revista Colombiana de Psiquiatría, 38 (3), 488-512.

Torkelson, E., y Muhonen, T. (2004). The role of gender and job level in coping with occupational stress. Work Stress, 18(3) ,267-274.

Trucco, M. (1998). Promoción de la salud mental en el ámbito laboral. Washington,D.C.: Organización Panamericana de la Salud. 